\title{
The Presence of Secondary Evans Syndrome in AIDS Patients with Talaromyces marneffei Infection
}

This article was published in the following Dove Press journal:

Infection and Drug Resistance

\author{
Mengyan Wang \\ Zhongdong Zhang \\ Jun Yan \\ Jinchuan Shi \\ Shourong Liu \\ Hu Wan
}

Department II of Infectious Diseases, Xixi Hospital of Hangzhou, Hangzhou Sixth People's Hospital, Hangzhou, 310023,

People's Republic of China
Correspondence: Hu Wan; Shourong Liu Xixi Hospital of Hangzhou, Hangzhou Sixth People's Hospital, Hangzhou, 310023 , People's Republic of China Tel + I35887| 0040

Email wanhul218@sina.com; Isr85463990@I26.com
Objective: Talaromyces marneffei (T.M) is a common opportunistic fungus in human immunodeficiency virus (HIV) infection individual. Secondary Evans syndrome in AIDS patients with Talaromyces marneffei infection has not been reported before. Here, we described cases related to this comorbidity.

Methods: AIDS patients diagnosed with Talaromyces marneffei infection from 2016 to 2020 at Xixi Hospital of Hangzhou were included in this retrospective study.

Results: Total 76 AIDS patients with T.M infection were enrolled. The most common symptoms were fever and cough (70/76; 55/76, respectively). 53/76 (69.74\%) patients got positive results of direct antiglobulin test. 14/76 AIDS-T.M patients combined with secondary Hemophagocytic lymphohistiocytosis (HLH). Five patients were diagnosed with AIDST.M associated Evans syndrome. There were severe inflammatory reaction, liver dysfunction, coagulation dysfunction and immunodeficiency status in AIDS-T.M patients with secondary Evans syndrome. All patients received antifungal therapy and three patients received corticosteroids for Evans syndrome treatment. One patient died due to sepsis.

Conclusion: AIDS-T.M patients with secondary Evans syndrome is extremely rare and we need to be alert to the occurrence of secondary Evans syndrome in AIDS-T.M patients. Clinicians should timely start effective antifungal treatments with suspicious T.M infection in AIDS patients.

Keywords: Talaromyces marneffei, Evans syndrome, human immunodeficiency virus, clinical characteristics

\section{Introduction}

Talaromyces marneffei (T.M), formerly known as Penicillium marneffei, a pathogenic thermally dimorphic fungus, can cause systemic mycosis in immunocompromised patients especially people living with human immunodeficiency virus (HIV). Talaromyces marneffei infection among HIV patients usually manifested as fever, cutaneous lesions, with low CD4 T-cell count and low hemoglobin level. ${ }^{1}$

In 1959, professor Gabriel Segretain formally described Talaromyces marneffei as a novel species. ${ }^{2}$ And bamboo rat is the reservoir for T. marneffei infection in humans. It can be a vector for human infections by acting as amplifiers of infectious dispersal stages. ${ }^{3}$ Previous studies had reported that $T$. marneffei infection is endemic in southeast Asia, India, Vietnam, Thailand and China. ${ }^{4-6}$ Moreover, a previous study had indicated that there are 50,000 HIV-positive individuals newly infected by T. marneffei every year in high-risk areas. ${ }^{7}$ And it is estimated that the mortality of T. marneffei-infected patients is 25.0 per 100 person-months in southern China. ${ }^{8}$ 
Evans syndrome (ES) has both immune thrombocytopenia (ITP) and autoimmune hemolytic anemia (AIHA), and $27.3 \%$ were classified as secondary Evans syndrome. ${ }^{9}$ The mortality had increased in secondary ES compared to primary ES and secondary ES is associated with haematological malignancies, autoimmune disorders (AID), infections, primary immunodeficiencies (PID). Blood smear, viral tests, serum protein electrophoresis, protein immunofixation and immunoglobulin concentrations, circulating lymphocyte phenotyping, flow cytometry for paroxysmal nocturnal haemoglobinuria clone detection, antinuclear antibodies and anti-dsDNA antibodies, lupus anticoagulant assay and antiphospholipid antibodies, bone marrow aspiration and karyotyping, bone marrow biopsy, CT scan of the chest, abdomen and pelvis, genetic explorations could help the diagnosis of secondary ES. ${ }^{10}$ Before the use of rituximab, the mortality rate of adults with both AIHA and Evans syndrome was $15 \%$ to $20 \%{ }^{11}$ Only two cases had been reported the presence of secondary Evans syndrome in HIV infection. ${ }^{12,13}$ There were no related researches which reported AIDS-T.M patients with secondary Evans syndrome.

In this study, we describe clinical characteristics of hospitalized AIDS-T.M patients and focus on AIDS-T.M associated Evans syndrome patients.

\section{Methods}

\section{Study Population}

This retrospective study included AIDS patients diagnosed with Talaromyces marneffei infection from January 1, 2016 to April 1, 2020 at Xixi Hospital of Hangzhou. Xixi Hospital of Hangzhou, located in Zhejiang Province, is one of the major infectious specialty tertiary teaching hospitals and is responsible for difficult and complicated HIV-infected cases assigned by the government.

Data extracted from the medical records included demographic information (sex and age), clinical characteristics (symptoms and signs), laboratory findings (a complete blood count, serum biochemistry, hemagglutination index, C-reactive protein, serum amyloid A, procalcitonin, specimen culture, lymphocyte subset analysis and blood gas analysis were conducted at admission), chest CT scan, treatment measures and clinical outcomes.

Inclusion criteria were as follows: (i) HIV-positive; (ii) a clear diagnosis of T.M infection. Patients with other immune deficiencies (including malignancy, congenital immunodeficiency, and receipt of chemotherapy); and immune reconstitution inflammatory syndrome (IRIS) were excluded. This study was approved by Ethics Committee of Xixi Hospital of Hangzhou in China. All procedures and methods were performed in accordance with the relevant international guidelines and regulations in order to reduce physical discomfort of the subjects. This study was conducted in accordance with the Declaration of Helsinki.

\section{Diagnostic Criteria}

T.M infection was diagnosed by a culture positive of T. marneffei from patients' specimens including sputum, alveolar lavage fluid, blood, bone marrow and lymph nodes. Cultures of clinical specimens were established on Sabouraud's dextrose agar at $25^{\circ} \mathrm{C}$ and $37^{\circ} \mathrm{C}$. HIV infection was confirmed in accordance with Centers for Disease Control and Prevention (CDC) definitions. History of AIDS diagnosis was determined according to HIV/AIDS management guidelines. ${ }^{14}$

Evans syndrome is a condition of AIHA accompanied by ITP. ITP diagnosis was based on a platelet count of $<10010^{\wedge}$ 9/L and exclusion of rapid onset thrombocytopenia not related to liver diseases (cirrhosis and portal hypertension), splenomegaly (haematological malignancies, Gaucher disease), drug-related thrombocytopenia, bone marrow deficiency (myelodysplastic syndromes, haematological malignancies, metastatic cancer) or inherited thrombocytopenia. ${ }^{10,15}$ AIHA diagnosis was based on a positive direct antiglobulin test associated to signs of hemolysis (hemoglobin reductions, reticulocytosis, unconjugated hyperbilirubinemia, elevated lactate dehydrogenase, and low haptoglobin). ${ }^{16}$ Bone marrow aspirate showed active erythroid hyperplasia, hemolysis and immature megakaryocytes, granular megakaryocytes (Figure 1A). Peripheral blood smear showed broken red blood cells (Figure 1B). Bone marrow analyses showed the absence of infections with MTB, MAC, Histoplasma, CMV and leukemia. In addition, clinicians had excluded the diagnosis of haematological malignancies, autoimmune disorders (AID) and primary immunodeficiencies (PID). According to the updated Hemophagocytic lymphohistiocytosis (HLH) 2004 criteria, ${ }^{17}$ diagnosis of HLH requires five of the following eight features: fever, splenomegaly, cytopenia affecting $\geq 2$ of 3 cell lineages (hemoglobin $<90 \mathrm{~g} / \mathrm{L}$; platelets $<100^{*} 10^{\wedge} 9 \mathrm{~L}$; and neutrophils $<1.0^{*} 10^{\wedge} 9 / \mathrm{L}$ ), hypertriglyceridemia and/or hypofibrinogenemia (fasting triglyceride $\geq 3 \mathrm{mmol} / \mathrm{L}$; fibrinogen $\leq 1.5$ $\mathrm{g} / \mathrm{L}$ ), hemophagocytosis, hyperferritinemia ( $\geq 500 \mathrm{mcg} / \mathrm{L}$ ), 


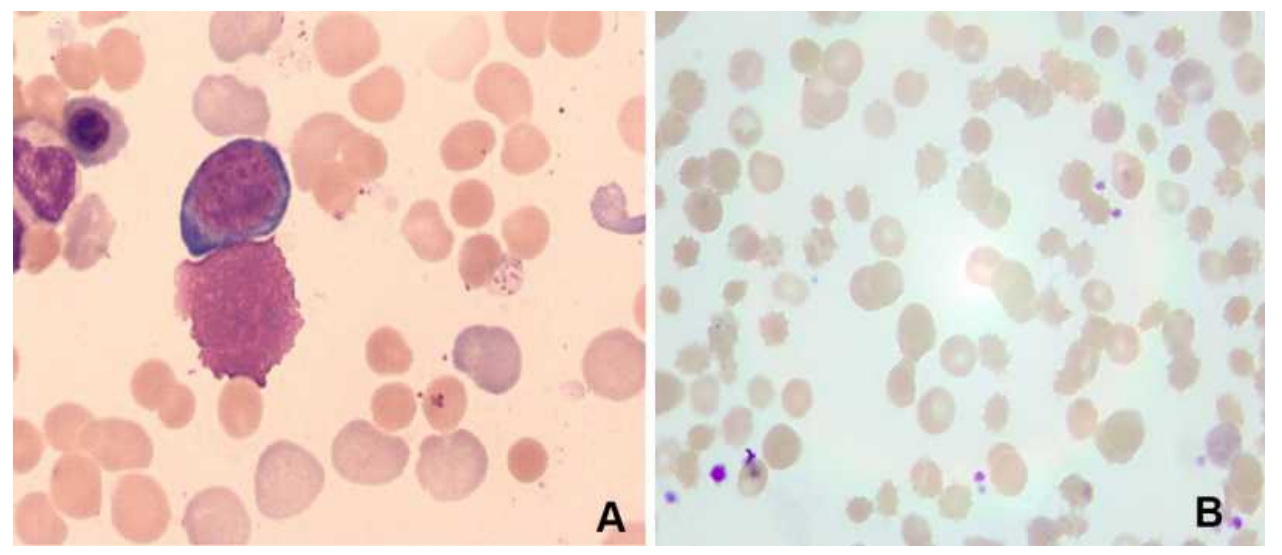

Figure I (A) Bone marrow smear showed that erythroid hyperplasia is obviously active, megakaryocytes are reduced, and the production of platelet is poor (magnification: 1000x). (B) The peripheral blood smear showed broken red blood cells (magnification: 100x).

high sIL-2R levels $(\geq 2400 \mathrm{U} / \mathrm{mL})$, and low/absent NK cell activity.

\section{Results}

\section{Characteristics of the Study Population}

Clinical characteristics and baseline demographics of the 76 AIDS-T.M patients are shown in Table 1. Male (72/76) were the majority of patients and the average age was 38.3 years old. The most common symptoms were fever and cough $(70 / 76 ; 55 / 76$, respectively). All patients received antifungal treatment after sampling. 53/76 (69.74\%) patients got positive results of direct antiglobulin test. 14/ 76 AIDS-T.M patients combined with secondary HLH. According to the diagnostic criteria, five patients were diagnosed with T.M-associated Evans syndrome. All patients had fever and three of them had HLH simultaneously. Among the five patients, three had overlapped other infection and three had not received highly active antiretroviral therapy (HARRT) before (Table 2).

\section{Laboratory Data of AIDS-T.M Associated Evans Syndrome Patients}

Inflammation indexes including $\mathrm{C}$-reactive protein and serum amyloid protein A concentrations were increased in all patients. The concentration of procalcitonin was increased in three patients. All patients revealed decreased lymphocyte counts, hemoglobin concentrations, platelet counts and increased reticulocyte counts. Two of them showed reduced white blood cell levels. These patients showed liver dysfunction. The concentration of aspartate aminotransferase was increased in all patients and the concentration of alanine aminotransferase concentration was increased in two patients. Four patients had increased prothrombin times and activated partial thromboplastin times. The median albumin level was $20.5 \mathrm{~g} / \mathrm{L}$ significantly below the normal range. All patients had significantly increased lactate dehydrogenase and D-dimer concentrations. Two of them revealed decreased fibrinogen concentrations. The median CD4+ T-cell counts were only $9 / \mu \mathrm{L}$. All patients showed significant reductions in CD4+ T-cell counts and two of them showed low level of CD8+ T-cell counts. Four patients showed low level of NK cell counts and three patients showed low level of B CD19+ cell counts (Table 3 ).

\section{Treatment and Outcomes of AIDS-T.M Associated Evans Syndrome Patients}

All patients received voriconazole as antifungal therapy when they were admitted to hospital according to our clinical assessment. Two patients changed to receive amphotericin B therapy after the diagnosis of T.M infection. And two weeks later, patient 2 started oral voriconazole $200 \mathrm{mg}$ Q12H and patient 3 started oral itraconazole 200mg Q12H for maintenance treatment. Patient 1 and patient 4 continued intravenous voriconazole therapy until two weeks then changed to oral for maintenance treatment. Patient 5 died due to sepsis when hospitalized for 3 days before the diagnosis of T.M infection. The remaining four patients had significant improvement of their clinical symptoms and left hospital with an average of 29 days (range: 17-45 days). Three patients received corticosteroids for Evans syndrome treatment (Table 4). After successful antifungal treatment, the phenomenon of Evans syndrome was disappeared. 
Table I Clinical Characteristics of AIDS-T.M Patients

\begin{tabular}{|c|c|}
\hline Characteristics & Patients $(n=76)$ \\
\hline Gender (M/F) & $72 / 4$ \\
\hline Age, years & $38.30 \pm 10.56$ \\
\hline \multicolumn{2}{|l|}{ HIV-RNA, IU/mL } \\
\hline$<100 \mathrm{IU} / \mathrm{mL}$ & $21(27.63 \%)$ \\
\hline$\geq 100 \mathrm{IU} / \mathrm{mL}$ & 55 (72.37\%) \\
\hline \multicolumn{2}{|l|}{ Clinical characteristics on admission } \\
\hline Body temperature, ${ }^{\circ} \mathrm{C}$ & $38.53 \pm 0.94$ \\
\hline Mean arterial pressure, $\mathrm{mmHg}$ & $123.06 \pm 17.93$ \\
\hline Respiratory rate, breaths/min & $19.78 \pm 2.11$ \\
\hline Heart rate, times/min & $109.96 \pm 18.60$ \\
\hline Skin lesions, no. (\%) & $26(34.21 \%)$ \\
\hline Respiratory symptoms, no. (\%) & $55(72.36 \%)$ \\
\hline \multicolumn{2}{|l|}{ Underline disease } \\
\hline Tuberculosis & $10(13.16 \%)$ \\
\hline Syphilis & $8(10.53 \%)$ \\
\hline CMV & II (14.47\%) \\
\hline EB & $3(3.95 \%)$ \\
\hline HBV & $4(5.26 \%)$ \\
\hline Candida & $25(32.89 \%)$ \\
\hline \multicolumn{2}{|l|}{ Treatment } \\
\hline Antifungal treatment, no. (\%) & $76(100 \%)$ \\
\hline HARRT before TM, no. (\%) & 31 (40.79\%) \\
\hline Glucocorticoid therapy, no. (\%) & $15(19.74 \%)$ \\
\hline Enlarged lymph nodes, no. (\%) & $52(68.42 \%)$ \\
\hline Enlarged liver and spleen, no. (\%) & $4 \mathrm{l}(53.95 \%)$ \\
\hline Positive of direct antiglobulin test, no. (\%) & $53(69.74 \%)$ \\
\hline Hemophagocytic lymphohistiocytosis, no. (\%) & $14(18.42 \%)$ \\
\hline Mortality, no. (\%) & $2(2.63 \%)$ \\
\hline
\end{tabular}

Abbreviations: $\mathrm{EBV}$, Epstein-Barr virus; $\mathrm{TB}$, tuberculosis; $\mathrm{HBV}$, hepatitis $B$ virus.

\section{Discussion}

A large sample size retrospective cohort study conducting by Jiang et al had indicated the prevalence of $T$. marneffe $i$ infections among HIV/AIDS inpatients was $16.1 \%$ that ranks fourth among all AIDS-related complications, after pneumonia, tuberculosis and oral candidiasis infections in southern China. ${ }^{8}$ In this study, we analyzed the characteristics and laboratory data in AIDS-T.M patients. The most common symptoms were fever and cough and were similar to the previous study. ${ }^{1}$ Importantly, we found that Evans syndrome was secondary to AIDS-T.M patients which was not be taken seriously before. Therefore, this is the first study reporting the clinical features of AIDS-T.M associated Evans syndrome.

Previous cases had reported the presence of Evans syndrome in HIV infection but had not described the AIDS-associated complications. ${ }^{12,13}$ The diagnosis of ES is ignored in AIDS-T.M individuals. And the pathogenesis of T.M infection associated with ES was still unclear. Previous research had indicated the causative factor of Evans syndrome may be associated with underlying deficiency in humoral or cell-mediated immunity. ${ }^{18}$ In our study, all patients had a real low level of CD4+ T-cell counts and two of them showed low level of CD8+ T-cell counts. Three patients showed low level of B CD19+ cell counts. Moreover, three of AIDS-T. M-associated Evans syndrome patients had HLH simultaneously. And the key factors of HLH are the imbalance of immunomodulation, accumulation of immunocompetent cells, and production of inflammatory cytokines. ${ }^{19}$ Pan et al had reported T.M infection secondary HLH in nine HIV-negative patients and suggested the

Table 2 Clinical Characteristics of AIDS-T.M Associated Evans Syndrome Patients

\begin{tabular}{|c|c|c|c|c|c|}
\hline Patient & Age/Sex & Underline Disease & Clinical Manifestations & HIV-RNA, IU/mL & HARRT \\
\hline PI & 29/M & No & Fever, skin lesion, hepatosplenomegaly & ND & No \\
\hline P2 & $26 / M$ & No & Fever, hepatosplenomegaly, weight loss & $<100$ & $\begin{array}{l}\text { I year } \\
\text { 3TC+TDF+EFV }\end{array}$ \\
\hline P3 & $47 / M$ & Candida & Fever & $4.44 * 10^{\wedge} 4$ & No \\
\hline P4 & $30 / M$ & TB, HBV & Fever, cough, skin lesion, hepatosplenomegaly & $1.45 * 10^{\wedge} 4$ & No \\
\hline P5 & $4 I / M$ & EBV & Fever & $<100$ & $\begin{array}{l}\text { I month } \\
3 T C+T D F+D T G\end{array}$ \\
\hline
\end{tabular}

Abbreviations: 3TC, lamivudine; TDF, tenofovir; EFV, efavirenz; DTG, dotegravir; ND, not done. 
Table 3 Laboratory Data of AIDS-T.M Associated Evans Syndrome Patients

\begin{tabular}{|c|c|c|c|c|c|c|c|}
\hline Variables & Normal Ranges & PI & P2 & P3 & P4 & P5 & Median (IQR) \\
\hline CRP, mg/L & $0-10$ & $121 \uparrow$ & $85 \uparrow$ & $68 \uparrow$ & $33 \uparrow$ & $127 \uparrow$ & $85(50.5-124)$ \\
\hline $\mathrm{PCT}, \mathrm{ng} / \mathrm{mL}$ & $0-0.500$ & $\mathrm{I} .03 \uparrow$ & $6.43 \uparrow$ & $0.812 \uparrow$ & $\mathrm{I} .4 \uparrow$ & $76.6 \uparrow$ & $1.4(0.92-4 \mid .5 I)$ \\
\hline $\mathrm{SAA}, \mathrm{mg} / \mathrm{L}$ & $0-10$ & $150 \uparrow$ & $\mid 12 \uparrow$ & $150 \uparrow$ & $5 I \uparrow$ & $150 \uparrow$ & $150(81.5-150)$ \\
\hline WBC, $10^{\wedge} 9 / \mathrm{L}$ & $3.50-9.50$ & $3.09 \downarrow$ & 7.68 & 6.4 & 4.57 & $3.46 \downarrow$ & $4.57(3.27-7.04)$ \\
\hline Reticulocyte, $10^{\wedge} \mid 2 / \mathrm{L}$ & $0.024-0.093$ & $0.18 \uparrow$ & $0.24 \uparrow$ & $0.15 \uparrow$ & $0.35 \uparrow$ & $0.11 \uparrow$ & $0.18(0.12-0.295)$ \\
\hline Neutrophil, I0^9/L & $1.80-6.30$ & 2.53 & $6.96 \uparrow$ & 6.15 & 3.78 & 2.7 & $3.78(2.61-6.55)$ \\
\hline Lymphocyte, I0^9/L & $1.10-3.20$ & $0.45 \downarrow$ & $0.62 \downarrow$ & $0.13 \downarrow$ & $0.44 \downarrow$ & $0.28 \downarrow$ & $0.44(0.205-0.535)$ \\
\hline $\mathrm{HGB}, \mathrm{g} / \mathrm{l}$ & $130-175$ & $109 \downarrow$ & $88 \downarrow$ & $109 \downarrow$ & $83 \downarrow$ & $86 \downarrow$ & $88(84.5-109)$ \\
\hline PLT, I0^9/L & $125-350$ & $85 \downarrow$ & $22 \downarrow$ & $71 \downarrow$ & $47 \downarrow$ & $38 \downarrow$ & $47(30-78)$ \\
\hline $\mathrm{LDH}, \mathrm{U} / \mathrm{L}$ & $120-250$ & $4084 \uparrow$ & $868 \uparrow$ & $442 \uparrow$ & $299 \uparrow$ & $577 \uparrow$ & $577(370.5-2476)$ \\
\hline Lactic acid, mmol/L & $0.7-2.1$ & $2.9 \uparrow$ & $9.61 \uparrow$ & 1.7 & $7.4 \uparrow$ & $2.8 \uparrow$ & $2.9(2.25-8.5)$ \\
\hline PT, S & $9.7-13.5$ & 13.4 & $14.6 \uparrow$ & $13.6 \uparrow$ & $17.2 \uparrow$ & $18.6 \uparrow$ & $14.6(\mid 3.5-17.9)$ \\
\hline APTT, S & $23.0-32.6$ & 31.9 & $42.3 \uparrow$ & $35.8 \uparrow$ & $35.8 \uparrow$ & $41.2 \uparrow$ & $35.8(33.85-41.75)$ \\
\hline $\mathrm{FIB}, \mathrm{g} / \mathrm{L}$ & $1.80-3.50$ & 2.23 & $0.96 \downarrow$ & 3.18 & $1.29 \downarrow$ & 2.44 & $2.23(\mathrm{I} .125-2.8 \mathrm{I})$ \\
\hline D-dimer, mg/L & $0.00-0.55$ & $77.39 \uparrow$ & $9.26 \uparrow$ & $4 I .4 I \uparrow$ & $2.5 \uparrow$ & $23.29 \uparrow$ & $23.29(5.88-59.4)$ \\
\hline Albumin, $g / L$ & $40.0-55.0$ & $29.3 \downarrow$ & $20.5 \downarrow$ & $20.2 \downarrow$ & $18.4 \downarrow$ & $21.3 \downarrow$ & $20.5(19.3-25.3)$ \\
\hline ALT, U/L & $7-40$ & $204 \uparrow$ & 28 & 23 & 21 & $92 \uparrow$ & $28(22-148)$ \\
\hline AST, U/L & $15-46$ & $767 \uparrow$ & $\mid 78 \uparrow$ & $66 \uparrow$ & $99 \uparrow$ & $230 \uparrow$ & $178(82.5-498.5)$ \\
\hline Creatinine, $\mu \mathrm{mol} / \mathrm{L}$ & $57-111$ & $47 \downarrow$ & $55 \downarrow$ & 58 & 58 & $147 \uparrow$ & $58(5 \mid-102.5)$ \\
\hline CD4+ T-cell counts, $/ \mu \mathrm{L}$ & $250-1561.6$ & $19 \downarrow$ & 77 $\downarrow$ & $I \downarrow$ & $5 \downarrow$ & $9 \downarrow$ & $9(3-48)$ \\
\hline CD8+ T-cell counts, $/ \mu \mathrm{L}$ & $203.5-1347.2$ & 307 & 747 & $75 \downarrow$ & 278 & $104 \downarrow$ & $278(89.5-527)$ \\
\hline NK T-cell counts, $/ \mu \mathrm{L}$ & $88-640$ & $13 \downarrow$ & $35 \downarrow$ & $28 \downarrow$ & $14 \downarrow$ & 139 & $28(13.5-87)$ \\
\hline B CDI9+ cell counts, $/ \mu \mathrm{L}$ & $77-736$ & 94 & $66 \downarrow$ & $21 \downarrow$ & 110 & $6 \downarrow$ & $66(13.5-102)$ \\
\hline
\end{tabular}

Notes: $\downarrow$ Lower than normal ranges. $\uparrow$ Higher than normal ranges.

Abbreviations: WBC, white cell count; HGB, hemoglobin; PLT, platelet; FIB, fibrinogen; LDH, lactate dehydrogenase; AST, aspartate aminotransferase; ALT, alanine aminotransferase; PT, prothrombin time; APTT, activated partial thromboplastin time; CRP, C-reactive protein; PCT, procalcitonin; SAA, serum amyloid protein A.

Table 4 Treatment of AIDS-T.M Associated Evans Syndrome Patients

\begin{tabular}{|c|c|c|c|}
\hline No. & Antifungal Therapy & ES Therapy & Outcome \\
\hline PI & $\begin{array}{l}\text { IV VCZ } 200 \mathrm{mg} \text { every } 12 \\
\mathrm{~h} \text { for two weeks then oral } \\
\text { VCZ } 200 \mathrm{mg} \text { Q } 12 \mathrm{H}\end{array}$ & MTH 40mg & Improve \\
\hline P2 & $\begin{array}{l}\text { IV Amphotericin B } 25 \mathrm{mg} / \\
\text { day for two weeks then } \\
\text { oral VCZ } 200 \mathrm{mg} \mathrm{Q} 12 \mathrm{H}\end{array}$ & $\begin{array}{l}\text { Dexamethasone } \\
5 \mathrm{mg}\end{array}$ & Improve \\
\hline P3 & $\begin{array}{l}\text { IV Amphotericin B } 25 \mathrm{~m} \mathrm{g/} \\
\text { day for two weeks oral ITC } \\
200 \mathrm{mg} \text { QI } 2 \mathrm{H}\end{array}$ & $\begin{array}{l}\text { Dexamethasone } \\
5 \mathrm{mg}\end{array}$ & Improve \\
\hline P4 & $\begin{array}{l}\text { IV VCZ } 200 \mathrm{mg} \text { every } 12 \\
\text { h for two weeks then oral } \\
\text { VCZ } 200 \mathrm{mg} \text { Q } 12 \mathrm{H}\end{array}$ & No & Improve \\
\hline P5 & $\begin{array}{l}\text { IV VCZ } 200 \mathrm{mg} \text { every } 12 \\
\mathrm{~h} \text { then oral VCZ } 200 \mathrm{mg} \\
\mathrm{Q} \mid 2 \mathrm{H}\end{array}$ & No & Death \\
\hline
\end{tabular}

Abbreviations: IV, intravenous; VCZ, voriconazole; ITC, itraconazole; MTH, methylprednisolone. pathogenesis of T.M infection associated with secondary HLH may involve severe inflammatory response syndrome caused by congenital or post-infection immune deficiency or by severe infection. ${ }^{20}$

AIDS-T.M associated secondary ES also involved severe inflammatory reaction, worse liver and coagulation function, worse immune status in our study. One patient died due to septic shock. Therefore, we think severe inflammatory response may increase the risk of death. In addition, the in-hospital mortality rate was lower (2/76) compared with previous studies (12-20\%). ${ }^{8,21,22}$ We suggest that the early antifungal treatment may reduce the mortality. All patients received antifungal treatment after sampling before diagnosed in our study. Two cases did not receive corticosteroids cure and one of them dead. Another patient has relatively weak inflammatory response, a real low level of CD4+ T-cell counts, relatively higher level of CD8+ T-cell counts and B CD19+ cell counts, and finally got improved. We think it may associate with 
inflammatory reaction and immune status. The small sample size also may cause deviations in mortality.

Our research has some limitations. First, this is a retrospective cohort study with all patients coming from one hospital, Xixi Hospital of Hangzhou, responsible for difficult and complicated HIV-infected cases. Second, we could not get the control cohort of HIV-negative $\mathrm{T}$. M-associated secondary ES.

\section{Conclusions}

AIDS-T.M patients with secondary Evans syndrome are extremely rare and we need to be alert to the occurrence of secondary Evans syndrome in AIDS-T.M patients. These patients are associated with a severe systematic status including severe inflammatory reaction, worse liver and coagulation function, worse immune status. Clinicians should timely start effective antifungal treatments with suspicious T.M infection in AIDS patients even if the results of T.M cultivation have not been obtained.

\section{Abbreviations}

ES, Evans syndrome; T.M, Talaromyces marneffei; HIV, human immunodeficiency virus; HLH, Hemophagocytic lymphohistiocytosis; ITP, immune thrombocytopenia; AIHA, autoimmune hemolytic anemia; SOFA, Sequential Organ Failure Assessment; MODS, Multiple Organ Dysfunction Score; TB, total bilirubin; WBC, white cell count; HGB, hemoglobin; PLT, platelet; FIB, fibrinogen; LDH, lactate dehydrogenase; ALT, alanine aminotransferase; PT, prothrombin time; APTT, activated partial thromboplastin time; CRP, C-reactive protein; PCT, procalcitonin.

\section{Data Sharing Statement}

The datasets used and/or analysed during the current study are available from the first author (Mengyan Wang, email: 21618054@zju.edu.cn) on reasonable request.

\section{Ethics Approval and Consent to Participate}

All patients provided written informed consent and this study was approved by Ethics Committee of Xixi Hospital of Hangzhou in China. All procedures and methods were performed in accordance with the relevant international guidelines and regulations in order to reduce physical discomfort of the subjects. This study was conducted in accordance with the Declaration of Helsinki.

\section{Acknowledgments}

This work was funded by Key projects of the Ministry of Science and Technology (No. 2018YFC1705701) and Provincial Natural Science Foundation (No. LS18H19001). The funding body had no role in the design of the study and collection, analysis, interpretation of data or preparation of the manuscript.

\section{Disclosure}

The authors declare that they have no competing interests.

\section{References}

1. Chen J, Zhang R, Shen Y, et al. Clinical characteristics and prognosis of penicilliosis among human immunodeficiency virus-infected patients in Eastern China. Am J Trop Med Hyg. 2017;96(6): 1350-1354. doi:10.4269/ajtmh.16-0521

2. Segretain G. Penicillium marneffei n. sp., agent d'une mycose du système réticulo-endothélial. Mycopathol Mycol Appl. 1959;11 (4):327-353. doi:10.1007/BF02089507

3. Cao C, Liang L, Wang W, et al. Common reservoirs for Penicillium marneffei infection in humans and rodents, China. Emerg Infect Dis. 2011;17(2):209-214. doi:10.3201/eid1702.100718

4. Vanittanakom N, Cooper CR, Fisher MC, Sirisanthana T. Penicillium marneffei infection and recent advances in the epidemiology and molecular biology aspects. Clin Microbiol Rev. 2006;19(1):95-110. doi:10.1128/CMR.19.1.95-110.2006

5. Cuong DD, Thorson A, Sönnerborg A, et al. Survival and causes of death among HIV-infected patients starting antiretroviral therapy in north-eastern Vietnam. Scand J Infect Dis. 2012;44(3):201-208. doi:10.3109/00365548.2011.631937

6. Wong SSY, Siau H, Yuen KY. Penicilliosis marneffei - West meets East. J Med Microbiol. 1999;48(11):973-975. doi:10.1099/00222 615-48-11-973

7. Armstrong-James D, Meintjes G, Brown GD. A neglected epidemic: fungal infections in HIV/AIDS. Trends Microbiol. 2014;22 (3):120-127. doi:10.1016/j.tim.2014.01.001

8. Jiang J, Meng S, Huang S, et al. Effects of Talaromyces marneffei infection on mortality of HIV/AIDS patients in southern China: a retrospective cohort study. Clin Microbiol Infect. 2019;25(2):233-241. doi:10.1016/j.cmi.2018. 04.018

9. Hansen DL, Möller S, Andersen K, Gaist D, Frederiksen H. Evans syndrome in adults - incidence, prevalence, and survival in a nationwide cohort. Am J Hematol. 2019;94(10):1081-1090. doi:10.1002/ajh.25574

10. Audia S, Grienay N, Mounier M, Michel M, Bonnotte B. Evans' syndrome: from diagnosis to treatment. J Clin Med. 2020;9(12):3851. doi: $10.3390 /$ jcm9123851

11. Michel M. [Characteristics of warm autoimmune hemolytic anemia and Evans syndrome in adults]. Presse Med. 2008;37(9):1309-1318. doi:10.1016/j.lpm.2008.01.026. French.

12. Sangle SA, Lohiy RV. Evan's syndrome in HIV infection. $J$ Assoc Physicians India. 2012;60:49-50.

13. Amid A, Leung E. Evans syndrome secondary to HIV infection. J Pediatr Hematol Oncol. 2013;35(6):490-491. doi:10.1097/MPH. 0b013e3182a05a93

14. Chinese Medical Association. [Chinese guidelines for diagnosis and treatment of HIV/AIDS (2018)]. Zhonghua Nei Ke Za Zhi. 2018;57(12):867-884. doi:10.3760/cma.j.issn.0578-1426.2018.12. 002. Chinese. 
15. Rodeghiero F, Stasi R, Gernsheimer T, et al. Standardization of terminology, definitions and outcome criteria in immune thrombocytopenic purpura of adults and children: report from an international working group. Blood. 2009;113(11):2386-2393. doi:10.1182/blood2008-07-162503

16. Bass GF, Tuscano ET, Tuscano JM. Diagnosis and classification of autoimmune hemolytic anemia. Autoimmun Rev. 2014;13(4-5):560-564. doi:10.1016/j.autrev.2013.11.010

17. Griffin G, Shenoi S, Hughes GC. Hemophagocytic lymphohistiocytosis: an update on pathogenesis, diagnosis, and therapy. Best Pract Res Clin Rheumatol. 2020;34:101515. doi:10.1016/j.berh.2020.101515

18. Dhingra KK, Jain D, Mandal S, Khurana N, Singh T, Gupta N. Evans syndrome: a study of six cases with review of literature. Hematology. 2008;13(6):356-360. doi:10.1179/102453308X343518

19. Egeler RM, Shapiro R, Loechelt B, Filipovich A. Characteristic immune abnormalities in hemophagocytic lymphohistiocytosis. J Pediatr Hematol Oncol. 1996;18(4):340-345. doi:10.1097/0004 3426-199611000-00002
20. Pan M, Qiu Y, Zeng W, et al. Talaromycosis-associated secondary hemophagocytic lymphohistiocytosis in nine human immunodeficiency virus-negative patients: a multicenter retrospective study. Infect Drug Resist. 2019;12:3807-3816. doi:10.2147/IDR. S232713

21. Kawila R, Chaiwarith R, Supparatpinyo K. Clinical and laboratory characteristics of penicilliosis marneffei among patients with and without HIV infection in Northern Thailand: a retrospective study. BMC Infect Dis. 2013;13:464. doi:10.1186/1471-2334-13464

22. Larsson M, Nguyen LH, Wertheim HF, et al. Clinical characteristics and outcome of Penicillium marneffei infection among HIV-infected patients in northern Vietnam. AIDS Res Ther. 2012;9(1):24. doi:10.1186/1742-6405-9-24
Infection and Drug Resistance

\section{Publish your work in this journal}

Infection and Drug Resistance is an international, peer-reviewed openaccess journal that focuses on the optimal treatment of infection (bacterial, fungal and viral) and the development and institution of preventive strategies to minimize the development and spread of resistance. The journal is specifically concerned with the epidemiology of
Dovepress

antibiotic resistance and the mechanisms of resistance development and diffusion in both hospitals and the community. The manuscript management system is completely online and includes a very quick and fair peerreview system, which is all easy to use. Visit http://www.dovepress.com/ testimonials.php to read real quotes from published authors. 